\section{Visual outcomes following ICG assisted ILM peel for Macular Hole}

\begin{abstract}
Purpose To evaluate the anatomical and visual outcome of indocyanine green (ICG)assisted internal limiting membrane (ILM) peeling for macular hole surgery. A literature search identified potential factors that may affect the functional success of the procedure. Methods Retrospective case note review of 14 consecutive patients undergoing phacovitrectomy, gas and ICG-assisted ILM peeling for macular hole from July 2001 to July 2002. A $0.5 \%$ ICG solution (osmolarity $270 \mathrm{mOsm}$ ) was left in contact with the retina for 1-3 min. The outcome measures were hole closure, change in visual acuity, and macular pigmentary changes.

Results Anatomical hole closure was achieved in 13 of 14 eyes (92.8\%). The mean logMAR Snellen acuities were 0.80 (range $0.60-1.30)$ preoperatively and $0.77(0.48-1.30)$ postoperatively. Seven eyes $(50 \%)$ developed retinal pigmentary changes in the macular area Conclusions ICG-assisted ILM peeling for macular hole surgery achieves high rates of anatomical hole closure, but functional results are less encouraging. Previous studies suggest toxicity of the ICG to the retina, at the level of the RPE or inner retina. The results may be optimized by using a lower concentration, isoosmolar, viscous solution, and by minimizing contact time of the solution and intensity, and duration of illumination.
\end{abstract}

Eye (2005) 19, 279-283. doi:10.1038/sj.eye.6701455

Published online 2 July 2004

Keywords: macular hole; ICG; ILM

\section{Introduction}

Peeling of the internal limiting membrane (ILM) of the retina during macular hole surgery may relieve tangential traction, and improve
D Posselt, R Rahman, M Smith and PR Simcock

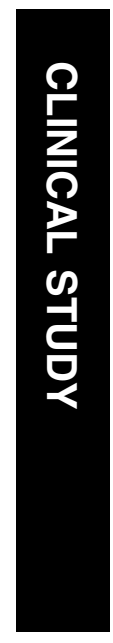

anatomical and functional outcomes. ${ }^{1}$ It is a technically demanding procedure, and many surgeons prefer to stain the membrane with indocyanine green (ICG) to provide contrast and facilitate peeling, resulting in high anatomical success rates. ${ }^{2-6}$ Recent clinical ${ }^{7,8}$ and histopathological ${ }^{9-11}$ studies have suggested that ICG may be toxic to the retina. We assessed anatomical and functional results of ICG-assisted ILM peel for macular hole and attempted to identify potential factors that may affect the outcome of the procedure through a search of the literature.

\section{Methods}

A case note review was performed on 14 consecutive patients who had undergone vitrectomy, gas injection, and ICG-assisted peeling of the ILM for macular hole at the Royal Devon and Exeter Hospital between July 2001 and July 2002.

Thirteen patients underwent combined phacovitrectomy with clear lens extraction at the time of surgery, using self-sealing clear corneal incisions. The phacoemulsification was performed prior to vitrectomy, but the intraocular lens was not placed in the eye until the end of surgery. The remaining patient was pseudophakic. A standard three-port pars plana vitrectomy was performed under general anaesthesia. If no posterior vitreous detachment was present, the posterior vitreous face was separated using the cutter on suction mode held close to the optic disc. ICG solution $(0.2-0.4 \mathrm{ml}$ of $0.5 \%$ ) was injected over the macula and left for 1-3 min before being aspirated. The ILM was then peeled in a circumferential manner around the macular hole to achieve an ILM defect of approximately two disc diameters.

Fluid-air exchange was followed by flushing with $50 \mathrm{ml}$ of $20 \% \mathrm{C}_{2} \mathrm{~F}_{6}$ gas prior to closure of the sclerostomies. Patients were advised to
West of England, Eye Unit Royal Devon and Exeter Hospital, UK

Correspondence: PR Simcock West of England Eye Unit Royal Devon and Exeter Hospital Barrack Road Exeter EX2 5DW, UK Tel: 0441392406008 Fax: 0441392406022 E-mail: psimcock@ hotmail.com

Received: 1 July 2003 Accepted: 12 January 2004 Published online: 2 July 2004

Previously presented as a poster at the Royal College Congress in Birmingham, May 2003 of Ophthalmologists Annual 
posture face down if the gas fill was less than $70 \%$, or if a stage 4 macular hole was present. If the gas fill was greater than $70 \%$, no posture was required other than to avoid lying on their back for 10 days. The technique of routine phacovitrectomy without face-down posture has been described in a previous study. ${ }^{12}$

To prepare the ICG solution, $25 \mathrm{mg}$ of sterile ICG powder was dissolved in $0.5 \mathrm{ml}$ of sterile aqueous solvent. This was diluted in $4.5 \mathrm{ml}$ of balanced salt solution, resulting in a $0.5 \%$ solution with an osmolarity of $270 \mathrm{mOsm}$. This concentration and method of preparation was advised by a facilitating consultant at an American Academy of Ophthalmology meeting in 2001.

Patients were reviewed at 2 weeks and 3 months after surgery (except for three patients, who were discharged after 2 months). Best-corrected visual acuities were recorded at discharge using a Snellen chart and converted to $\log$ MAR format. Patients had been refracted by an optometrist for final visual acuity measurement.

\section{Results}

Eight holes were stage 2, two stage 3, and three stage 4 . A total of 12 patients were female, and the average age was 71 years (range 62-78 years). The mean duration of the macular hole prior to surgery was 7 months (range 2-18 months). ICG exposure time was $3 \mathrm{~min}$ for nine cases and $1 \mathrm{~min}$ for the other five. Three patients developed significant posterior capsular opacification requiring YAG capsulotomy.

\section{Anatomical results}

Of the 14 holes, 13 were flat and closed on follow-up examination, giving an anatomical success rate of $92.8 \%$. The other hole remained elevated and open. Seven eyes (50\%) developed macular pigmentary changes (Figures 3 and 4). No macular oedema was seen clinically following surgery with the fundal contact lens.

\section{Functional results}

The mean preoperative acuity was 0.80 . (range $0.60-1.30$ ). The mean postoperative acuity was 0.77 (range 0.48 1.30). Of the 14 eyes, one improved by one line of Snellen visual acuity, three improved by two lines, and one improved by three lines. Three eyes were unchanged and six eyes reduced by 1 line of acuity (Figure 1 ). Of the seven eyes with macular pigment changes, three eyes reduced by one line of acuity, three remained the same, and one improved by two lines.

The patients whose retinae were exposed to the ICG solution for only $1 \mathrm{~min}$ appeared to experience better functional outcomes ( $80 \%$ of patients had improved visual acuity) than those exposed for $3 \mathrm{~min}$, of whom
$11.1 \%$ improved (by only one line of acuity), $33.3 \%$ were unchanged, and $55.5 \%$ worsened (Figure 2). In addition, there was a higher rate of macular RPE changes in the group exposed for $3 \mathrm{~min}$ (six out of nine or $66.7 \%$, compared with $20 \%$ for the group exposed for $1 \mathrm{~min}$ ).

\section{Discussion}

Tangential traction is believed to initiate macular holes, and may originate from collagen and cellular elements on either side of the ILM. The enlargement is thought to be due to myofibroblastic contraction on the ILM. ${ }^{1}$ The meta-analysis by Mester and Kuhn ${ }^{1}$ suggests that ILM peeling yields an anatomical success rate of $96 \%$ and a functional success rate (improvement of two or more lines of Snellen acuity) of $81 \%$. This compares with figures of $77 \%$ and $55 \%$, respectively, for surgery without ILM peeling from the same study.

ICG has been shown to selectively stain the ILM, ${ }^{2}$ and therefore facilitates identification of this very thin, transparent membrane for easier removal. ${ }^{2-6}$ However, recent studies have suggested less favourable functional results, and histopathologic analysis of operative ${ }^{9}$ and cell culture ${ }^{10,11}$ specimens has suggested that ICG may be toxic to the retina. Our observations during this study support these findings.

Toxic effects of ICG may have increased significance as animal studies have suggested that it may pass along the length of the optic nerve by axonal transport after intravitreal injection. ${ }^{13}$ In addition, persistent fluorescence may be detected at the macula 3 months, and at the optic nerve 6 months after surgery. ${ }^{14}$

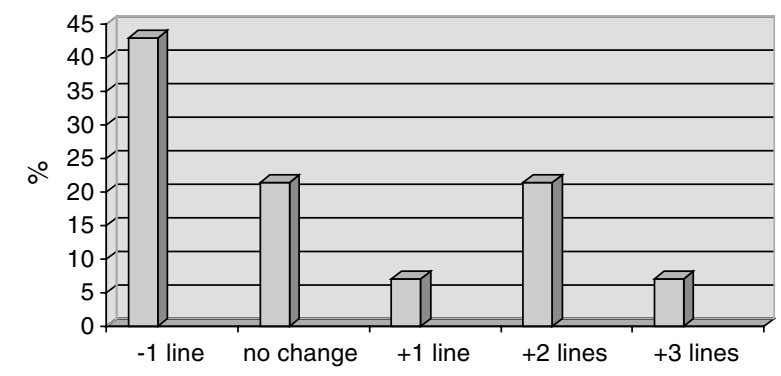

Figure 1 Functional results.

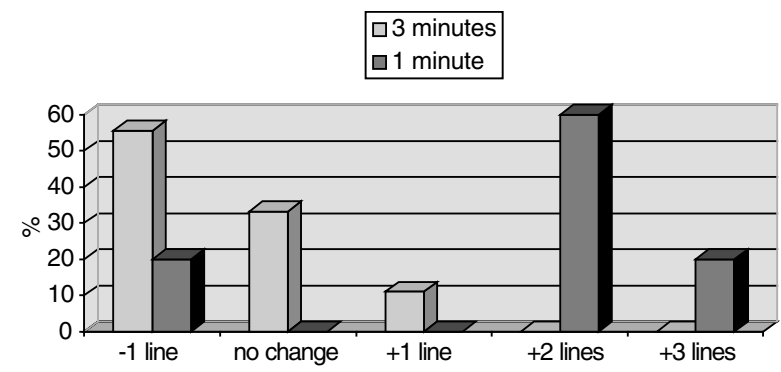

Figure 2 Functional results by duration of ICG exposure. 
Table 1 Outcomes according to concentration of ICG used

\begin{tabular}{lccccc}
\hline Study & $N$ & Conc Used & Anatomical Outcome & Functional Success Rate $^{\mathrm{a}}$ & Other \\
\hline Current study & 14 & $0.5 \%$ & $92.8 \%(13 / 14)$ & $28.6 \%$ & \\
Kandosono $\mathrm{et}^{4}$ & 13 & $0.06 \%$ in viscomaterial & $92 \%(12 / 13)$ & $84 \%$ & $47.6 \%$ had macular RPE atrophy \\
Engelbrecht $\mathrm{el}^{7}$ & 21 & $0.1 \%$ & $86 \%(18 / 21)$ & $23.8 \%$ & $70 \%$ \\
Kwok $\mathrm{et}^{6}$ & 10 & $0.025 \%$ & $100 \%$ & \\
\hline
\end{tabular}

${ }^{\mathrm{a}}$ Functional success defined as improvement by two or more lines of Snellen acuity.

\section{Possible basis for adverse effects of ICG}

Toxic effects of ICG on the retinal pigment epithelium (RPE) Evidence for ICG toxicity to the RPE has been seen in clinical and cell culture findings. Engelbrecht $e^{2} \mathrm{al}^{7}$ noted atrophic RPE changes in the area of the macular hole in 10 out of 21 eyes (47.6\%). This is comparable with our findings of macular pigmentary changes in seven out of 14 eyes (50\%). A much lower incidence of macular pigmentary changes $(5.7 \%)$ was reported in a series of 105 eyes undergoing ILM peeling without ICG by Haritoglou et al..$^{15}$ The postulated causes for this included impaired choroidal circulation due to gas tamponade, light toxicity, mechanical trauma, and suction of the subretinal fluid through the macular hole.

Studies involving RPE cell cultures have also demonstrated ICG toxicity. Increased cell death ${ }^{11}$ and reduced mitochondrial enzyme activity ${ }^{10}$ have been demonstrated in cultures exposed to ICG compared with those exposed to control solutions such as phosphatebuffered solution (PBS) or balanced salt solution (BSS). These changes relate to the clinical findings, as ICG comes in direct contact with the RPE through the macular hole during staining.

Induced damage to innermost retinal layers Ultrastructural analysis (by electron microscopy) of ILM specimens removed during ICG-assisted surgery has revealed adherent cell membranes and even entire Muller cell foot processes, as well as other unidentified cell debris. ${ }^{8,9}$ This is consistent with the subjective findings during this study that the ILM behaved differently and felt 'thicker' during peeling compared to surgery without ICG, suggesting a deeper cleavage plane.

Similar damage to the nerve fibre layer may explain the visual field defects reported by Haritoglou et $a l^{8}$ in $35 \%$ of eyes following ICG-assisted ILM peel, compared with no observed field defects in eyes undergoing unassisted ILM peel. However, other studies have reported small field defects following unassisted ILM peel, ${ }^{15}$ and also following macular hole surgery without ILM peel. ${ }^{1}$ Other possible causes include dehydration of nerve fibres during air-fluid exchange, ${ }^{1}$ trauma to the nerve fibre layer during ILM peel, ${ }^{8}$ and trauma to the

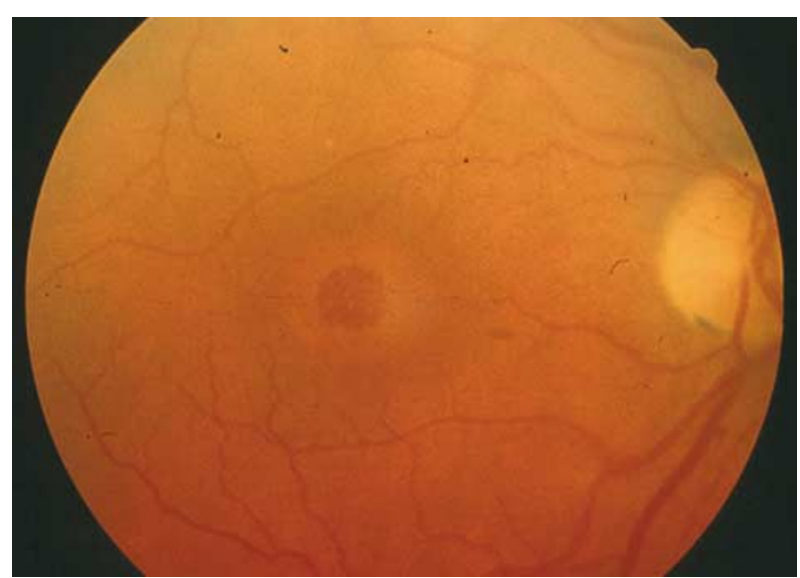

Figure 3 Preoperative stage 3 full-thickness macular hole.

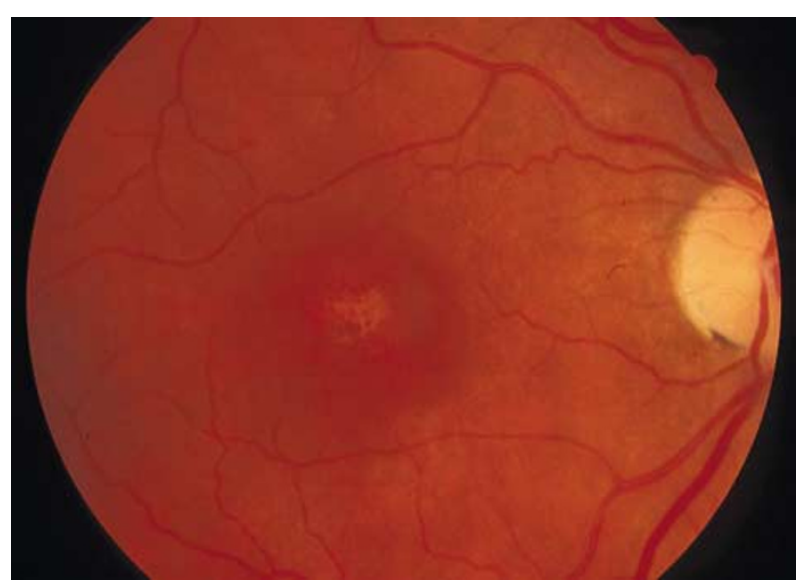

Figure 4 Postoperative appearance with hole closure but pigmentary changes present.

optic nerve head during induced posterior vitreous detachment.

\section{Technical factors that may alter adverse outcomes}

Concentration of ICG used Concentration may be the main factor influencing outcome of ICG-assisted ILM peel. ${ }^{7,9}$ Concentrations seen in studies have varied by more than a factor of 20. Analysis of these studies suggests a correlation between higher concentrations and adverse outcomes, as shown in Table 1. 
Duration of ICG exposure prior to removal If the adverse results are assumed to be due to toxicity of the ICG to the retina, it would seem logical that duration of exposure should also have an influence on the outcome. This is supported by Engelbrecht $e t a l^{7}$ who maintained a concentration of $0.1 \%$, but varied the duration of exposure from $30 \mathrm{sec}$ to $2 \frac{1}{2} \mathrm{~min}$. While there was only a limited number of articipants in the study, there would seem to be a trend towards poorer functional outcome with longer exposures (Table 2).

These findings were supported by the current study, in which the results among eyes exposed for only $1 \mathrm{~min}$ were superior to those among eyes exposed for $3 \mathrm{~min}$.

Further evidence of the toxicity of ICG can be seen by comparing outcomes with the product of concentration and time, as in Table 3.

Viscosity of the solution Kandonosono et al ${ }^{4}$ used a viscomaterial to make up a $0.06 \%$ solution of ICG. This may be of importance, given the previous concern regarding direct contact of the solution with the RPE through the macular hole, which may be reduced or prevented by a more viscous solution.

Osmolarity of the solution Stalmans et al ${ }^{11}$ demonstrated significantly increased cell death among cultured RPE cells exposed to hypo-osmolar solutions (including ICG) compared with cell cultures exposed to iso-osmolar (294-314 mOsm) solutions such as PBS. An analysis of other studies ${ }^{2,6-8}$ reveals a correlation between functional

Table 2 Outcomes according to duration of ICG exposure (Engelbrecht $e t a l^{7}$ )

\begin{tabular}{|c|c|c|}
\hline Exposure Time & Rate of RPE atrophy & Functional outcome \\
\hline 30 s (4 eyes) & 1 of 4 & $75 \%$ better; $25 \%$ worse \\
\hline $1 \min (3)$ & 2 of 3 & $\begin{array}{l}33 \% \text { better; } 33 \% \text { same } \\
33 \% \text { worse }\end{array}$ \\
\hline $2 \min (13)$ & 7 of 13 & $\begin{array}{l}7.7 \% \text { better; } 23.1 \% \text { same; } \\
69.2 \% \text { worse }\end{array}$ \\
\hline $2.5 \min (1)$ & 0 of 1 & No change \\
\hline
\end{tabular}

outcome and osmolarity of solution used, with those using iso-osmolar solutions generally reporting better results. The exception was Kandonosono et al, ${ }^{4}$ who obtained good results with a hypo-osmolar solution $(270 \mathrm{mOsm})$, but this had the possible aforementioned protective effect of viscosity.

Increased phototoxicity Engelbrecht et al ${ }^{7}$ suggests that ICG may reduce the 'safe time' for light exposure to the macula. They suggest that this may be avoidable by reducing the light intensity during ILM peeling, and by ensuring a safe distance between the light source and the retina.

\section{Conclusion}

While ICG staining may facilitate ILM peeling during macular hole surgery, legitimate concerns have been raised regarding adverse functional outcomes from this technique. These concerns are supported by clinical and pathological findings following ICG use, including our present study. Further studies are required to determine whether a safe and effective technique of ICG staining of the ILM is possible through optimization of identified factors such as concentration, exposure time, phototoxicity, viscosity, and osmolarity of the solution.

\section{References}

1 Mester V, Kuhn F. Internal limiting membrane removal in the management of full-thickness macular holes. Am J Ophthalmol 2000; 129: 769-777.

2 Gandorfer A, Messmer EM, Ulbig MW, Kampik A. Indocyanine green selectively stains the internal limiting membrane. Am J Ophthalmol 2001; 131: 387-388.

3 Kusaka S, Hayashi N, Ohji M, Hayashi A, Kamei M, Tano Y. Indocyanine green facilitates removal of epiretinal and internal limiting membranes in myopic eyes with retinal detachment. Am J Ophthalmol 2001; 131: 388-390.

4 Kandonosono K, Itoh N, Uchio E, Nakamura S, Ohno S. Staining of internal limiting membrane in macular hole surgery. Arch Ophthalmol 2000; 118: 1116-1118.

Table 3 Outcomes according to product of concentration and time

\begin{tabular}{lccl}
\hline Study & $N$ & Conc. $\times$ Time (min.\%) & Functional Outcome \\
\hline Current study & 9 & 1.5 & $11.1 \%$ better; $33.3 \%$ same; $55.6 \%$ worse \\
& 5 & 0.5 & $80 \%$ better; 20\% worse \\
Kandosono $\mathrm{et}^{4}$ & 13 & 0.03 & $84 \%$ better (rest unchanged) \\
Engelbrecht $\mathrm{et}^{7}$ & 21 & 0.05 & $75 \%$ better; $25 \%$ worse \\
& & 0.1 & $33 \%$ better; $33 \%$ same; $33 \%$ worse \\
& & 0.2 & $7.7 \%$ better; $23.1 \%$ same; $69.2 \%$ worse \\
Kwok et $^{7}$ & 10 & 0.25 & No change \\
& & 0.0125 & $70 \%$ better (rest unchanged)
\end{tabular}


5 Burk SE, Da Mata AP, Snyder ME, Rosa RH, Foster RE. Indocyanine green-assisted peeling of the retinal internal limiting membrane. Ophthalmology 2000; 107: 2010-2014.

6 Kwok AKH, Li WWY, Pang CP, Lai TYY, Yam GHF, Chan NR et al. Indocyanine green staining and removal of internal limiting membrane in macular hole surgery: histology and outcome. Am J Ophthalmol 2001; 132: 178-183.

7 Engelbrecht NE, Freeman J, Sternberg P, Aaberg Sr TM, Aaberg Jr TM, Martin DF et al. Retinal pigment epithelial changes after macular hole surgery with indocyanine greenassisted internal limiting membrane peeling. Am J Ophthalmol 2002; 133: 89-94.

8 Haritoglou C, Gandorfer A, Gass CA, Schaumberger M, Ulbig MW, Kampik A. The effect of indocyanine green on functional outcome of macular pucker surgery. Am J Ophthalmol 2003; 135: 328-337.

9 Gandorfer A, Haritoglou C, Gass CA, Ulbig MW, Kampik A. Indocyanine green-assisted peeling of the internal limiting membrane may cause retinal damage. Am J Ophthalmol 2001; 132: 431-433.
10 Sippy BD, Engelbrecht NE, Hubbard GB, Moriarty SE, Jiang $\mathrm{S}$, Aaberg Jr TM et al. Indocyanine green effect on cultured human retinal pigment epithelial cells: implication for macular hole surgery. Am J Ophthalmol 2001; 132: 433-435.

11 Stalmans P, Van Aken EH, Veckeneer M, Feron EJ, Stalmans I. Toxic effect of indocyanine green on retinal pigment epithelium related to osmotic effects of the solvent. Am J Ophthalmol 2002; 134: 282-285.

12 Simcock PR, Scalia S. Phacovitrectomy without prone posture for full thickness macular holes. $\mathrm{Br}$ J Ophthalmol 2001; 85: 1316-1319.

13 Paques M, Genevois O, Regnier A, Tadayoni R, Secombe R, Gaudric A et al. Axon-tracing properties of indocyanine green. Arch Ophthalmol 2003; 121: 367-370.

14 Tadayoni R, Paques M, Girmens JF, Massin P, Gaudric A. Persistence of fundus fluorescence after use of indocyanine green for macular surgery. Ophthalmology 2003; 110: 604-608.

15 Haritoglou C, Gass CA, Schaumberger M, Ehrt O, Gandorfer A, Kampik A. Macular changes after peeling of the internal limiting membrane in macular hole surgery. Am J Ophthalmol 2001; 132: 363-368. 